\title{
Robust cooperative control of multiple heterogeneous negative-imaginary systems
}

DOI:

10.1016/j.automatica.2015.07.028

\section{Document Version}

Accepted author manuscript

Link to publication record in Manchester Research Explorer

\section{Citation for published version (APA):}

Wang, J., Lanzon, A., \& Petersen, I. R. (2015). Robust cooperative control of multiple heterogeneous negativeimaginary systems. Automatica, 61, 64-72. https://doi.org/10.1016/j.automatica.2015.07.028

\section{Published in:}

Automatica

\section{Citing this paper}

Please note that where the full-text provided on Manchester Research Explorer is the Author Accepted Manuscript or Proof version this may differ from the final Published version. If citing, it is advised that you check and use the publisher's definitive version.

\section{General rights}

Copyright and moral rights for the publications made accessible in the Research Explorer are retained by the authors and/or other copyright owners and it is a condition of accessing publications that users recognise and abide by the legal requirements associated with these rights.

\section{Takedown policy}

If you believe that this document breaches copyright please refer to the University of Manchester's Takedown Procedures [http://man.ac.uk/04Y6Bo] or contact uml.scholarlycommunications@manchester.ac.uk providing relevant details, so we can investigate your claim.

\section{OPEN ACCESS}




\title{
Robust Cooperative Control of Multiple Heterogeneous Negative-Imaginary Systems *
}

\author{
Jianan Wang ${ }^{\mathrm{a}}$, Alexander Lanzon ${ }^{\mathrm{b}}$, Ian R. Petersen ${ }^{\mathrm{c}}$ \\ ${ }^{a}$ Key Laboratory of Dynamics and Control of Flight Vehicle within Ministry of Education, School of Aerospace Engineering, \\ Beijing Institute of Technology, Beijing 100081, China \\ ${ }^{\mathrm{b}}$ Control Systems Centre, School of Electrical and Electronic Engineering, University of Manchester, Manchester M13 9PL, UK \\ ${ }^{\mathrm{c}}$ School of Information Technology and Electrical Engineering, University of New South Wales, Australian Defence Force \\ Academy, Canberra, A.C.T. 2600, Australia
}

\begin{abstract}
This paper presents a consensus-based robust cooperative control framework for a wide class of linear time-invariant (LTI) systems, namely Negative-Imaginary (NI) systems. Output feedback, dynamic, Strictly Negative-Imaginary (SNI) controllers are applied in positive feedback to heterogeneous multi-input-multi-output (MIMO) plants through the network topology to achieve robust output feedback consensus. Robustness to external disturbances and model uncertainty is guaranteed via NI system theory. Cooperative tracking control of networked NI systems is presented as an corollary of the derived results by adapting the proposed consensus algorithm. Numerical examples are also given to demonstrate the effectiveness of proposed robust cooperative control framework.
\end{abstract}

Key words: Cooperative control; Consensus; Robust control; Heterogeneous systems; Negative-Imaginary systems.

\section{Nomenclature}

$I_{n}$

$\mathbf{1}_{n}$

$M>(\geq) 0$

$M<(<) 0$

$\operatorname{Ker}(M)$

$\operatorname{Im}(M)$

$\operatorname{rank}(M)$

$\lambda_{i}(M), \bar{\lambda}(M)$ the $i$ th, largest eigenvalue of $M$

$\lambda(M), \operatorname{det}(M)$ spectrum, determinant of matrix $M$

$M^{T} \quad$ transpose of matrix $M$

$M^{*} \quad$ complex conjugate transpose of matrix $M$

$\mathbb{R}^{m \times n}, \mathbb{C}^{m \times n} \quad$ set of $m \times n$ real, complex matrices

$\operatorname{Re}[s] \quad$ real part of $s \in \mathbb{C}$

$\mathfrak{L}_{2} \quad$ abbreviation for $\mathfrak{L}_{2}[0, \infty)$

$\operatorname{Im}_{\mathfrak{L}_{2}}(G) \quad$ image of system $G(s)$ under all $\mathfrak{L}_{2}$ inputs

$\mathcal{R H}_{\infty} \quad$ set of real-rational stable transfer functions

$\left[P(s), P_{s}(s)\right]$ positive feedback interconnection of 2 plants

\footnotetext{
* This paper was not presented at any IFAC meeting. Corresponding author J. N. Wang. Tel. +86-1068911921

Email addresses: wangjianan@ieee.org (Jianan Wang), alexander.lanzon@manchester.ac.uk (Alexander Lanzon), i.r.petersen@gmail.com (Ian R. Petersen).
}

\section{Introduction}

Cooperative control of heterogeneous LTI systems has been widely studied in the literature and there is now a wealth of methods to handle different aspects of the nominal cooperative control problem. Robust cooperative control is however less studied due to the inherent complexities associated with robustness. For example, [2] studies a cooperative control problem for a string of coupled heterogeneous subsystems. Such systems can arise in vehicle platoons. However, the systems considered are constrained to SISO systems (due to the mathematics of the continued fractions used) and do not allow poles on the imaginary axis, and also the graph is only restricted to string connections. On the other hand, [17] solves a cooperative robust output regulation problem for a class of LTI systems with minimum phase dynamics. A combination of simultaneous high-gain state feedback control and a distributed high-gain observer is adopted to achieve cooperative output regulation under particular parameter uncertainty as well as particular external disturbances. From a different perspective, [22] discusses a full-state feedback robust consensus protocol for heterogeneous second-order multi-agent systems. Existing published literature on robust cooperative con- 
trol of heterogeneous multi-agent systems is hence restricted to either only SISO plants, or minimum phase LTI plants or full-state feedback second order plants.

NI systems theory has drawn much attention (e.g. $[4,12,19])$ since it was introduced in [7]. This is because there are a wide class of LTI systems with negative imaginary frequency response, for which applications can be easily found in a variety of fields including aerospace, large space structures, multi-link robotic arms usually with co-located position sensors and force actuators [13] and nano-positioning [9], etc. Also the NI systems class is invariant to additive NI model uncertainty and other type interconnections as discussed in [3]. Thus, result based on NI systems theory immediately yield robustness to spill-over dynamics $[7,13,16]$.

A square, real, rational, proper transfer function matrix $P(s)$ is NI if the following conditions are satisfied $([7,20,10]):(1) P(s)$ has no pole in $\operatorname{Re}[s]>0 ;(2) \forall \omega>0$ such that $j \omega$ is not a pole of $P(s), j\left(P(j \omega)-P(j \omega)^{*}\right) \geq$ 0 ; (3) If $s=j \omega_{0}$ where $\omega_{0}>0$ is a pole of $P(s)$, then it is a simple pole and the residue matrix $K=\lim _{s \rightarrow j \omega_{0}}(s-$ $\left.j \omega_{0}\right) j P(s)$ is Hermitian and positive semi-definite; (4) If $s=0$ is a pole of $P(s)$, then $\lim _{s \rightarrow 0} s^{k} P(s)=0 \forall k \geq 3$ and $P_{2}=\lim _{s \rightarrow 0} s^{2} P(s)$ is Hermitian and positive semi-definite. This definition includes free body dynamics which leads to dynamical models with poles at the origin, such as $\frac{s^{2}+1}{s^{2}\left(s^{2}+2\right)}$. Examples of NI systems can be found in $[10,13]$, and these include a single-integrator system, a doubleintegrator system, second-order systems such as those that arise in undamped and damped flexible structures or inertial systems, to name a few typically considered in the consensus literature. Cooperative control of multiple NI systems arise with the development of NI systems' applications where one single NI system is incapable of achieving the mission goals, for example, the load is too heavy to be carried by one multi-link robotic arm.

This paper solves the general problem, robust output feedback cooperative control of heterogeneous MIMO NI systems (possibly with poles on the imaginary axis) under external disturbances and model uncertainty. Unlike the literature, we impose no minimum phase assumption; the communication graph can be any general undirected and connected graph rather than any specific graph; we allow MIMO agents; we consider explicitly robustness to both unmodelled dynamics of arbitrary order and energy-bounded disturbances; we handle output feedback rather than full state feedback; and explicitly characterise a family of control laws that could be tuned for performance. Toward this end, NI system theory is adopted to first derive conditions for robust output feedback consensus and then transport the proposed results to cooperative tracking to obtain a robust output feedback cooperative control framework for a wide class of LTI systems.

Preliminaries of graph theory: $\mathcal{G}=(\mathcal{V}, \mathcal{E})$ where
$\mathcal{V}=\left\{v_{1}, v_{2}, \ldots, v_{n}\right\}$ and $\mathcal{E} \subseteq \mathcal{V} \times \mathcal{V}$ mathematically describes a graph with $n$ nodes and $l$ edges. An undirected and connected graph requires that there exists at least one bidirectional path in $\mathcal{E}$ connecting all nodes in $\mathcal{V}$. The incidence matrix $\mathcal{Q}$ of $\mathcal{G}$ is a $|\mathcal{V}| \times|\mathcal{E}|(n \times l)$ matrix, which can be attained by first letting each edge in the graph have an arbitrary but fixed orientation and then

$\mathcal{Q}:= \begin{cases}q_{v e}=1 & \text { if } v \text { is the initial vertex of edge } e \\ q_{v e}=-1 & \text { if } v \text { is the terminal vertex of edge } e, \\ q_{v e}=0 & \text { if } v \text { is not connected to } e\end{cases}$

For an undirected graph $\mathcal{G}, \mathcal{Q}$ is not unique but the corresponding Laplacian matrix is unique and given by $\mathcal{L}_{n}=\mathcal{Q} \mathcal{Q}^{T}$. Similarly, the edge-weighted Laplacian is also unique given by $\mathcal{L}_{e}=\mathcal{Q} \mathcal{K} \mathcal{Q}^{T}$, where $\mathcal{K} \geq 0$ is the diagonal edge weighting matrix. It is also shown in [1] that $\operatorname{rank}(\mathcal{Q})=n-1=\operatorname{rank}\left(\mathcal{L}_{n}\right)$ when $\mathcal{G}$ is connected and $\operatorname{rank}(\mathcal{Q})=n-1=\operatorname{rank}\left(\mathcal{L}_{e}\right)$ when $\mathcal{G}$ is connected and $\operatorname{det}(\mathcal{K}) \neq 0$. It is well-known [14] that $\mathcal{L}_{n}$ and $\mathcal{L}_{e}$ will both have one unique zero eigenvalue associated with the eigenvector $\mathbf{1}_{n}$ and all the other eigenvalues are positive and real, when $\operatorname{det}(\mathcal{K}) \neq 0, \mathcal{G}$ is undirected and connected. In this case, $\mathcal{L}_{n} \geq 0, \mathcal{L}_{e} \geq 0$, and

$$
\operatorname{Ker}\left(\mathcal{L}_{n}\right)=\operatorname{Ker}\left(\mathcal{L}_{e}\right)=\operatorname{Ker}\left(\mathcal{Q}^{T}\right)=\operatorname{span}\left\{\mathbf{1}_{n}\right\}
$$

Note also that, for an undirected and connected graph $\mathcal{G}$, any row removal of $\mathcal{Q}$ or column removal of $\mathcal{Q}^{T}$ yields a full row rank $\mathcal{Q}$ or a full column rank $\mathcal{Q}^{T}$ respectively by inspecting the relation of Laplacian matrix with $\mathcal{Q}$ and the property of positive semi-definite matrices with a kernel dimension of 1 [5].

\section{Robust Output Feedback Consensus}

In this section, we will consider robust output feedback consensus for multiple heterogeneous NI systems under $\mathfrak{L}_{2}$ external disturbance and additive SNI model uncertainty (as would arise in spill-over dynamics for truncated order flexible structures). Two cases will be discussed to cover all the heterogeneous cases. First of al1, let us begin with the problem formulation with the following notation: $\max _{i=1}^{n}\left\{a_{i}\right\}$ is the maximum value of $a_{i}, i \in\{1, \cdots, n\}$ and $\operatorname{diag}_{i=1}^{n}\left\{A_{i}\right\}$ is a block-diagonal matrix with $A_{i}, i \in\{1, \cdots, n\}$ on the diagonal. A square, real, rational, proper transfer function matrix $P_{s}(s)$ is SNI if the following conditions are satisfied: $(1) P_{s}(s)$ has no pole in $\operatorname{Re}[s] \geq 0 ;(2) \forall \omega>0, j\left(P_{s}(j \omega)-P_{s}(j \omega)^{*}\right)>0$. Examples of SNI systems include $\frac{1}{s+a}$ where $a>0$, $\frac{a}{s^{2}+b s+c}$ where $a, b, c>0$ or non-minimum phase systems such as $\frac{1-s}{2+s}$. See $[7,13]$ for further examples.

For multiple heterogeneous NI systems (in general MIMO) with $n>1$ agents, the transfer function of agent 
$i \in\{1, \cdots, n\}$ is given as

$$
\hat{\boldsymbol{y}}_{i}=\hat{P}_{i}(s) \hat{\boldsymbol{u}}_{i},
$$

where $\hat{\boldsymbol{y}}_{i} \in \mathbb{R}^{m_{i} \times 1}$ and $\hat{\boldsymbol{u}}_{i} \in \mathbb{R}^{m_{i} \times 1}$ are the output and input of agent $i$ respectively. In order to deal with the consensus of different dimensional inputs/outputs, $\hat{P}_{i}(s)$ can be padded with zeros up to $m=\max _{i=1}^{n}\left\{m_{i}\right\}$ and the locations of padding zeros depend on which output needs to be coordinated, for instance, $P_{i}(s)=\left[\begin{array}{cc}\hat{P}_{i}(s) & 0 \\ 0 & 0\end{array}\right]$ has dimension of $m$ such that the first $m_{i}$ outputs are to be coordinated, or $P_{i}(s)=\left[\begin{array}{cc}0 & 0 \\ 0 & \hat{P}_{i}(s)\end{array}\right]$ also has dimension of $m$, but now the last $m_{i}$ outputs are to be coordinated instead. Accordingly, the input $\hat{\boldsymbol{u}}_{i}$ and output $\hat{\boldsymbol{y}}_{i}$ are extended to be $\boldsymbol{u}_{i}=\left[\begin{array}{ll}\hat{\boldsymbol{u}}_{i}^{T} & 0\end{array}\right]^{T}$ or $\left[\begin{array}{ll}0 & \hat{\boldsymbol{u}}_{i}^{T}\end{array}\right]^{T} \in \mathbb{R}^{m \times 1}$, and $\boldsymbol{y}_{i}=\left[\begin{array}{ll}\hat{\boldsymbol{y}}_{i}^{T} & 0\end{array}\right]^{T}$ or $\left[\begin{array}{ll}0 & \hat{\boldsymbol{y}}_{i}^{T}\end{array}\right]^{T} \in \mathbb{R}^{m \times 1}$, respectively. Note that interleaving zero rows and corresponding columns within $\hat{P}_{i}(s)$ is also permissible. It can be easily seen that the above manipulation would preserve the NI property by checking the definition. Therefore, without loss of generality, the overall plant can be described as Fig. 1:

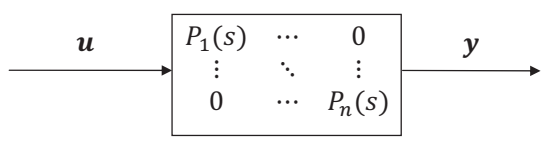

Fig. 1. Multiple Heterogeneous NI Plants

where $\boldsymbol{y}=\left[\boldsymbol{y}_{1}^{T}, \cdots, \boldsymbol{y}_{n}^{T}\right]^{T} \in \mathbb{R}^{n m \times 1}$ and $\boldsymbol{u}=$ $\left[\boldsymbol{u}_{1}^{T}, \cdots, \boldsymbol{u}_{n}^{T}\right]^{T} \in \mathbb{R}^{n m \times 1}$. We now define robust output feedback consensus as follows:

Definition 1 A distributed output feedback control law achieves robust output feedback consensus for a network of systems if for a family of plant dynamics and for all $\mathfrak{L}_{2}[0, \infty)$ disturbances on the plant input and/or plant output, $\boldsymbol{y}_{i}-\boldsymbol{y}_{\text {ss }} \in \mathfrak{L}_{2}[0, \infty) \forall i \in\{1, \cdots, n\}$. Here $\boldsymbol{y}_{\text {ss }}$ is the final convergence trajectory, which can be a function of time depending on the plant and controller dynamics.

Remark 2 Note that since transfer functions in $\mathcal{R} \mathcal{H}_{\infty}$ map $\mathfrak{L}_{2}[0, \infty)$ to $\mathfrak{L}_{2}[0, \infty)$ and due to the superposition theorem of linear systems [21], if there are no disturbances, then $\boldsymbol{y}_{i}-\boldsymbol{y}_{s s} \rightarrow \mathbf{0} \forall i \in\{1, \cdots, n\}$ in Definition 1 retrieving the typical consensus meaning in the literature.

Observe that if one were to construct the overall networked plant dynamics involving the heterogeneous multiple agents $P_{i}(s)$ and the communication- s graph represented by a Laplacian matrix $\mathcal{L}_{n}$ as $\left(\mathcal{L}_{n} \otimes I_{m}\right) \cdot \operatorname{diag}_{i=1}^{n}\left\{P_{i}(s)\right\}$, then the overall networked plant is not NI any more due to asymmetry despite each heterogeneous agent being individually NI. This would then make NI systems theory inapplicable. Instead, we can utilize the incidence matrix $\mathcal{Q}$ instead of $\mathcal{L}_{n}$ to reformulate the overall networked plant as shown in Fig. 2:

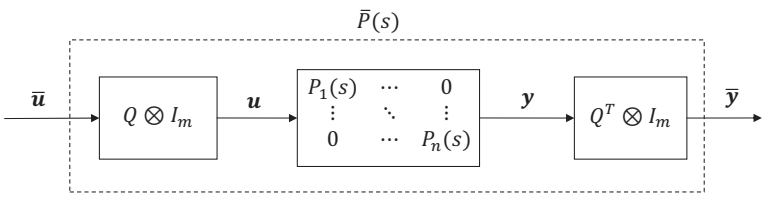

Fig. 2. Overall Network Plant

The reason for adopting the incidence matrix $\mathcal{Q}$ before and after the plant dynamics instead of a Laplacian matrix $\mathcal{L}_{n}=\mathcal{Q} \mathcal{Q}^{T}$ totally before or after the plant dynamics is to guarantee that the resultant controller is distributed and only uses local information. This will be explained in more detail later in this section. The augmented system can be derived as

$$
\overline{\boldsymbol{y}}=\bar{P}(s) \overline{\boldsymbol{u}}=\left(\mathcal{Q}^{T} \otimes I_{m}\right) \operatorname{diag}_{i=1}^{n}\left\{P_{i}(s)\right\}\left(\mathcal{Q} \otimes I_{m}\right) \overline{\boldsymbol{u}}
$$

where $\overline{\boldsymbol{y}}=\left[\overline{\boldsymbol{y}}_{1}^{T}, \cdots, \overline{\boldsymbol{y}}_{l}^{T}\right]^{T} \in \mathbb{R}^{l m \times 1}$ and $\overline{\boldsymbol{u}}=$ $\left[\overline{\boldsymbol{u}}_{1}^{T}, \cdots, \overline{\boldsymbol{u}}_{l}^{T}\right]^{T} \in \mathbb{R}^{l m \times 1}$ are the output and input vectors for the overall system. It can be concluded that the overall system $\bar{P}(s)$ is still NI due to the following lemmas:

Lemma $3 \underset{i=1}{\operatorname{diag}}\left\{P_{i}(s)\right\}$ is NI if and only if $P_{i}(s)$ are all $N I \forall i \in\{1, \cdots, n\}$.

The proof of Lemma 3 is straightforward from the definition of NI systems. The same argument also applies for SNI functions. The following lemma which is also straightforward from the definition of NI systems allows further manipulation.

Lemma 4 Given any NI MIMO $P(s)$, then $\bar{P}(s)=$ $F P(s) F^{*}$ is NI for any constant matrix $F$.

The output $\boldsymbol{y} \in \mathbb{R}^{n m \times 1}$ reaches consensus (i.e. $\boldsymbol{y}_{i}=\boldsymbol{y}_{j}$ $\forall i, j$ ) when $\overline{\boldsymbol{y}} \rightarrow \mathbf{0} \in \mathbb{R}^{l m \times 1}$ by noticing the properties of the incidence matrix $\mathcal{Q}$ given in (1). This formulation converts the output consensus problem to an equivalent internal stability problem. Then robustness properties can be studied via standard control theoretic methods to yield robust consensus results. We now impose the following assumptions throughout the rest of this paper:

Assumption $\mathbf{5} \mathcal{G}$ is undirected and connected.

Assumption 6 Let $\Delta_{i}(s) \forall i \in\{1, \cdots, n\}$ be arbitrary 
SNI systems satisfying $\bar{\lambda}\left(\Delta_{i}(0)\right)<\mu, \Delta_{i}(\infty)=0 \forall i \in$ $\{1, \cdots, n\}$, and $0<\mu \in \mathbb{R}$.

In the sequel, robust output feedback consensus will be discussed along two directions: NI plants without or with free body dynamics to cover all the heterogeneous types of NI systems.

\subsection{NI plants without free body dynamics}

In this subsection, NI plants without free body dynamics will be firstly considered, which also means $\hat{P}_{i}(s)$ has no poles at the origin. The following lemmas are needed:

$\underset{\bar{\lambda}}{\text { Lemma }} 7$ ([5]) Given $M \in \mathbb{R}^{n \times m}, \bar{\lambda}\left(M M^{T}\right)=$ $\bar{\lambda}\left(M^{T} M\right)$.

Lemma 8 Assume $M$ is Hermitian with $\bar{\lambda}(M) \geq 0$ and $N \geq 0$, we have $\bar{\lambda}(M N) \leq \bar{\lambda}(M) \bar{\lambda}(N)$.

PROOF. Since $M \leq \bar{\lambda}(M) I$, we obtain $N^{\frac{1}{2}} M N^{\frac{1}{2}} \leq$ $\bar{\lambda}(M) N$. With the conditon of $\bar{\lambda}(M) \geq 0, N^{\frac{1}{2}} M N^{\frac{1}{2}} \leq$ $\bar{\lambda}(M) N \leq \bar{\lambda}(M) \bar{\lambda}(N) I$. Thus, $\bar{\lambda}(M N)=\bar{\lambda}\left(N^{\frac{1}{2}} M N^{\frac{1}{2}}\right) \leq$ $\bar{\lambda}(M) \bar{\lambda}(\bar{N})$.

Lemma 9 ([7,20]) Given an NI transfer function $P(s)$ and an SNI function $P_{s}(s)$ with $P(s)$ having no pole $(s)$ at the origin, $P(\infty) P_{s}(\infty)=0$ and $P_{s}(\infty) \geq 0$. $\left[P(s), P_{s}(s)\right]$ is internally stable if and only if $\bar{\lambda}\left(P(0) P_{s}(0)\right)<1$.

Next we present the first main result of this paper with the definition of $\bar{P}_{s}(s)=\operatorname{diag}_{j=1}^{l}\left\{P_{s, j}(s)\right\}$ where $P_{s, j}(s)$ are arbitrary SNI compensators.

Theorem 10 Given a graph $\mathcal{G}$ with incidence matrix $\mathcal{Q}$, satisfying Assumption 5 and modelling the communication links among multiple NI agents $\hat{P}_{i}(s)$ with no pole(s) at the origin which are appropriately padded with rows and columns of zeros to give $P_{i}(s)$ in Fig. 3. Robust output feedback consensus is achieved via the output feedback control law

$$
\boldsymbol{u}=\left(\mathcal{Q} \otimes I_{m}\right) \bar{P}_{s}(s)\left(\mathcal{Q}^{T} \otimes I_{m}\right) \boldsymbol{y}
$$

(or in a distributed manner for agent $i$ via

$$
\boldsymbol{u}_{i}=\sum_{k=1}^{n} a_{i k} P_{s, j}(s)\left(\boldsymbol{y}_{i}-\boldsymbol{y}_{k}\right)
$$

where $a_{i k}$ are the elements of the adjacency matrix ${ }^{1}$ and $j$ is the edge connecting vertex $i$ to vertex $k$ ) under any external disturbances $w_{1} \in \operatorname{Im}_{\mathfrak{L}_{2}}\left(\mathcal{Q} \otimes I_{m}\right)$ and $w_{2} \in \mathfrak{L}_{2}$ if $\exists i \in\{1, \cdots, n\}: \bar{\lambda}\left(P_{i}(0)\right) \geq 0$ and $\forall i \in\{1, \cdots, n\}, j \in$

\footnotetext{
${ }^{1}$ See [14] for definition
}

$\{1, \cdots, l\}$ all the following conditions hold:

$$
\bar{\lambda}\left(P_{i}(0)\right) \bar{\lambda}\left(P_{s, j}(0)\right)<\frac{1}{\bar{\lambda}\left(\mathcal{L}_{n}\right)},
$$

$P_{i}(\infty) P_{s, j}(\infty)=0$ (where $i$ is the vertex of edge $j$ ) and $P_{s, j}(\infty) \geq 0$. The output feedback consensus control law (4) will be robust to all model uncertainty $\Delta_{i}(s), i \in$ $\{1, \cdots, n\}$ satisfying Assumption 6 if the D.C. gain of the SNI compensator $\bar{P}_{s}(s)$ is tuned more stringently such that $\forall i \in\{1, \cdots, n\}, j \in\{1, \cdots, l\}$

$$
\bar{\lambda}\left(P_{i}(0)\right)+\mu<\frac{1}{\bar{\lambda}\left(\mathcal{L}_{n}\right) \bar{\lambda}\left(P_{s, j}(0)\right)} .
$$

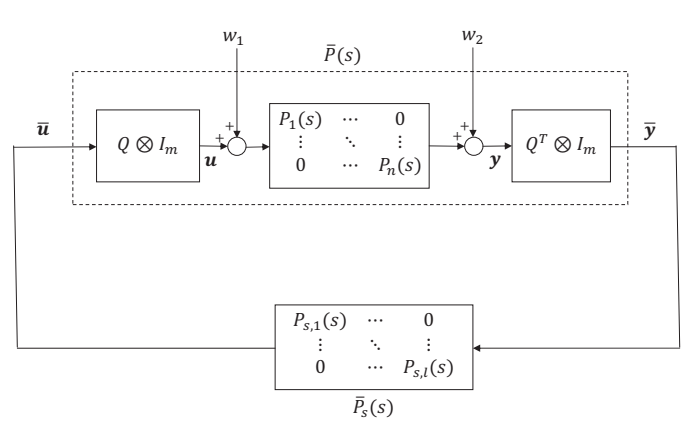

Fig. 3. Positive feedback interconnection with SNI compensators through the network topology

PROOF. From Fig. 3, Lemmas 3 and 4, it can be seen that $\bar{P}(s)$ is NI without pole(s) at the origin and $\bar{P}_{s}(s)$ is SNI. Applying Lemma 8, we obtain

$$
\begin{aligned}
& \bar{\lambda}\left(\bar{P}(0) \bar{P}_{s}(0)\right) \\
= & \bar{\lambda}\left(\left(\mathcal{Q}^{T} \otimes I_{m}\right) \operatorname{diag}_{i=1}^{n}\left\{P_{i}(0)\right\}\left(\mathcal{Q} \otimes I_{m}\right) \operatorname{diag}_{j=1}^{l}\left\{P_{s, j}(0)\right\}\right) \\
\leq & \bar{\lambda}\left(\left(\mathcal{Q}^{T} \otimes I_{m}\right) \operatorname{diag}_{i=1}^{n}\left\{P_{i}(0)\right\}\left(\mathcal{Q} \otimes I_{m}\right)\right) \max _{j=1}^{l}\left\{\bar{\lambda}\left(P_{s, j}(0)\right)\right\} \\
\leq & \max _{i=1}^{n}\left\{\bar{\lambda}\left(P_{i}(0)\right)\right\} \bar{\lambda}\left(\mathcal{Q}^{T} \mathcal{Q}\right) \max _{j=1}^{l}\left\{\bar{\lambda}\left(P_{s, j}(0)\right)\right\} \quad\left(\text { since } \exists i: \bar{\lambda}\left(P_{i}(0)\right) \geq 0\right) \\
= & \max _{i=1}^{n}\left\{\bar{\lambda}\left(P_{i}(0)\right)\right\} \max _{j=1}^{l}\left\{\bar{\lambda}\left(P_{s, j}(0)\right)\right\} \bar{\lambda}\left(\mathcal{L}_{n}\right) \quad(\text { by Lemma 7) }
\end{aligned}
$$

since $\bar{\lambda}(\bar{P}(0)) \geq 0$ (because $\exists i: \bar{\lambda}\left(P_{i}(0)\right) \geq 0$ ) and $P_{s, j}(0)>P_{s, j}(\infty) \geq 0 \forall j \in\{1, \cdots, l\}$ (due to Lemma 2 in [7] with the assumption of $\left.P_{s, j}(\infty) \geq 0\right)$. Thus, since $\exists i \in\{1, \cdots, n\}: \bar{\lambda}\left(P_{i}(0)\right) \geq 0$ and $\forall i=1, \cdots, n$ and $j=1, \cdots, l$, all of the following hold: $\bar{\lambda}\left(P_{i}(0)\right) \bar{\lambda}\left(P_{s, j}(0)\right)<\frac{1}{\lambda\left(\mathcal{L}_{n}\right)}, P_{i}(\infty) P_{s, j}(\infty)=0$ (where $i$ is the vertex of edge $j$ ) and $P_{s, j}(\infty) \geq 0,\left[\bar{P}(s), \bar{P}_{s}(s)\right]$ is internally stable via NI systems theory in Lemma 9. This then implies nominal output consensus when the disturbances $\boldsymbol{w}_{1}$ and $\boldsymbol{w}_{2}$ are set to zero by noting that $\overline{\boldsymbol{y}} \rightarrow \mathbf{0} \Leftrightarrow \boldsymbol{y}-\mathbf{1}_{n} \otimes \boldsymbol{y}_{s s} \rightarrow \mathbf{0}$, i.e., $\boldsymbol{y}_{i}-\boldsymbol{y}_{s s} \rightarrow \mathbf{0}$ since the graph $\mathcal{G}$ is undirected and connected. 
In addition, internal stability of $\left[\bar{P}(s), \bar{P}_{s}(s)\right]$ and superposition principle of linear systems $([21])$ guarantee that $\boldsymbol{y}_{i} \rightarrow \boldsymbol{y}_{s s}+\delta$ with $\delta \in \mathfrak{L}_{2}$ for all $\mathfrak{L}_{2}$ exogenous signal injections perturbing signals $\overline{\boldsymbol{u}}$ and $\overline{\boldsymbol{y}}$, which in turn means that any $w_{1} \in \operatorname{Im}_{\mathfrak{L}_{2}}\left(\mathcal{Q} \otimes I_{m}\right)$ and any $w_{2} \in \mathfrak{L}_{2}$ can be injected in Fig. 3. Hence, the control protocol (4) will achieve a perturbed $\mathfrak{L}_{2}$ consensus signal on output $\boldsymbol{y}$ (due to superposition principle of linear systems) for all disturbances $w_{1} \in \operatorname{Im}_{\mathfrak{L}_{2}}\left(\mathcal{Q} \otimes I_{m}\right)$ and $w_{2} \in \mathfrak{L}_{2}$.

Additive model uncertainties $\Delta_{i}(s) \forall i \in\{1, \cdots, n\}$ satisfying Assumption 6 can be dealt with as in [16], which is shown in Fig. 4.

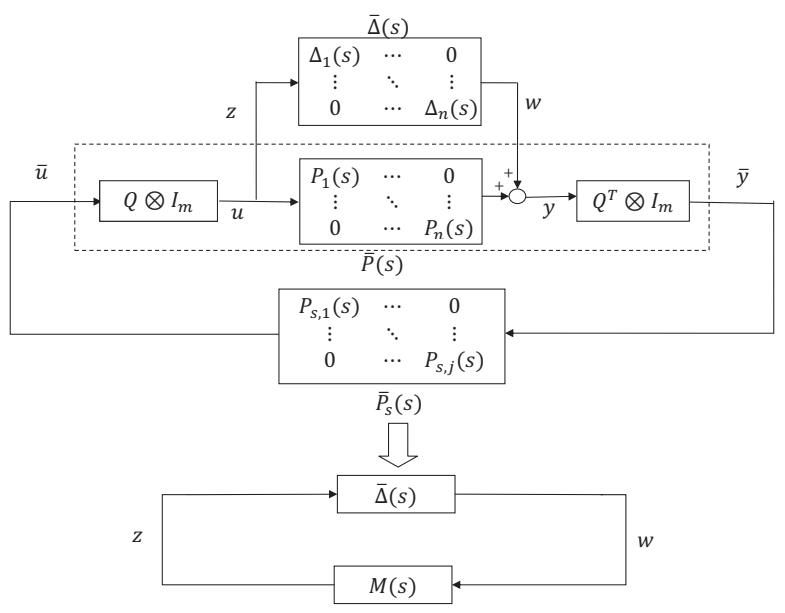

Fig. 4. Robustness to model uncertainty via NI system theory

Fig. 4 (top) can be manipulated to Fig. 4 (bottom) with $M(s)=\left(\mathcal{Q} \otimes I_{m}\right) \bar{P}_{s}(s)\left(I-\bar{P}(s) \bar{P}_{s}(s)\right)^{-1}\left(\mathcal{Q}^{T} \otimes I_{m}\right)$. Internal stability already yields $M(s) \in \mathcal{R} \mathcal{H}_{\infty}$ and $M(s)$ is NI via Theorem 6 in [3] in general, or via Theorem 6 in [13] when $P_{i}(s)$ have no poles on the imaginary axis. This NI system $M(s)$ is connected with $\bar{\Delta}(s)$ which fulfills Assumption 6. Now

$$
\begin{aligned}
& \bar{\lambda}(\Delta(0) M(0)) \\
\leq & \bar{\lambda}(\Delta(0)) \bar{\lambda}\left[\left(\mathcal{Q} \otimes I_{m}\right) \bar{P}_{s}(0)\left(I-\bar{P}(0) \bar{P}_{s}(0)\right)^{-1}\left(\mathcal{Q}^{T} \otimes I_{m}\right)\right] \\
\leq & \mu \bar{\lambda}\left(\mathcal{L}_{n}\right) \bar{\lambda}\left[\bar{P}_{s}(0)\left(I-\bar{P}(0) \bar{P}_{s}(0)\right)^{-1}\right] \\
\leq & \frac{\mu \bar{\lambda}\left(\mathcal{L}_{n}\right) \bar{\lambda}\left(\bar{P}_{s}(0)\right)}{1-\bar{\lambda}\left(\bar{P}(0) \bar{P}_{s}(0)\right)} \\
\leq & \frac{\mu \bar{\lambda}\left(\mathcal{L}_{n}\right) \max _{j=1}^{l}\left\{\bar{\lambda}\left(P_{s, j}(0)\right)\right\}}{1-\max _{i=1}^{n}\left\{\bar{\lambda}\left(P_{i}(0)\right)\right\} \bar{\lambda}\left(\mathcal{L}_{n}\right) \max _{j=1}^{l}\left\{\bar{\lambda}\left(P_{s, j}(0)\right)\right\}}
\end{aligned}
$$

It is then clear that inequality $(7)$ guarantees $\bar{\lambda}(\Delta(0) M(0))<$ 1 which in turn implies robust stability for all uncertainties that satisfy Assumption 6 .

Remark 11 Inequality (6) only provides a sufficient condition due to the heterogeneousness of systems. It also implies $\bar{\lambda}\left(P_{i}(0) P_{s, j}(0)\right) \leq \bar{\lambda}\left(P_{i}(0)\right) \bar{\lambda}\left(P_{s, j}(0)\right)<\frac{1}{\lambda\left(\mathcal{L}_{n}\right)}$ due to Lemma 8, which gives a more stringent condition than that of internal stability of $\left[P_{i}(s), P_{s, j}(s)\right]$, i.e., $\bar{\lambda}\left(P_{i}(0) P_{s, j}(0)\right)<1$ (on noting that $\bar{\lambda}\left(\mathcal{L}_{n}\right)>1$ due to [6]). This coincides with engineering intuition since the stability condition for networked systems is always more stringent than that of single agent system ([8]).

Remark 12 Since we assume that $\exists i: \bar{\lambda}\left(P_{i}(0)\right) \geq 0$ and since values of $i$ such that $\bar{\lambda}\left(P_{i}(0)\right) \leq 0$ automatically fulfill inequality (6), only values of $i$ such that $\bar{\lambda}\left(P_{i}(0)\right)>0$ need to be checked. For values of $i$ such that $\bar{\lambda}\left(P_{i}(0)\right)>0$, the D.C. gain of the SNI controllers always need to be tuned for small eigenvalues in order to satisfy inequality (6). SNI control synthesis for robust performance is beyond the scope of this paper. Interested readers are referred to $[15,16]$.

Remark 13 There is clearly a huge class of permissible dynamic perturbations to the nominal dynamics as Assumption 6 only imposes a restriction on $\Delta_{i}(s)$ at the frequency $\omega=0$ and $\omega=\infty$ and the SNI class has no gain (as long as it is finite gain) or order restriction [7]. The result in Theorem 10 is for additive perturbations, but similar analysis can be performed for other types of perturbations that preserve the NI class. A few examples of permissible perturbations that preserve the NI class include additive perturbations where uncertainty is also NI [7], feedback perturbations where both systems in the feedback interconnection are NI [13] and more general perturbations based Redheffer Star-products and Linear Fractional Transformations [3]. For example, $\frac{1}{s+5}$ and $\frac{\left(2 s^{2}+s+1\right)}{\left(s^{2}+2 s+5\right)(s+1)(2 s+1)}$ are SNI with the same D.C. gain.

\subsection{NI plants with free body dynamics}

In this subsection, we will consider more general NI plants by including free body dynamics (i.e. poles at the origin) under the assumption of strict properness, i.e. $P_{i}(\infty)=0$. Hence, this subsection covers the cases where the NI plant has poles at the origin. The NI class restricts the number of such poles at the origin to be at most 2. The following residue matrices carrying information about the properties of the free body motion for the NI system $\boldsymbol{y}=P(s) \boldsymbol{u}$ where $P(s) \in \mathbb{R}^{m \times m}$ ([10]): $P_{2}=\lim _{s \rightarrow 0} s^{2} P(s), P_{1}=\lim _{s \rightarrow 0} s\left(P(s)-\frac{P_{2}}{s^{2}}\right), P_{0}=$ $\lim _{s \rightarrow 0}\left(P(s)-\frac{P_{2}}{s^{2}}-\frac{P_{1}}{s}\right)$. It can be observed that $P_{1}=$ $0, P_{2}=0$ means there is no free body dynamics, $P_{1} \neq$ $0, P_{2}=0$ means there is free body dynamics with 1 pole at the origin, $P_{2} \neq 0$ means there is free body dynamics with 2 poles at the origin. Then, we can define the Hankel matrix $\Gamma$ as $\Gamma=\left[\begin{array}{cc}P_{1} & P_{2} \\ P_{2} & 0\end{array}\right] \in \mathbb{R}^{2 m \times 2 m}$, where $P_{1}, P_{2} \in \mathbb{R}^{m \times m}$. In the subsection, we assume $\Gamma \neq 0$ since either $P_{1} \neq 0$ or $P_{2} \neq 0$. Then $\Gamma$ can be decomposed by Singular Value Decomposition (SVD) as

$\Gamma=\left[\begin{array}{ll}U_{1} & U_{2}\end{array}\right]\left[\begin{array}{ll}S & 0 \\ 0 & 0\end{array}\right]\left[\begin{array}{c}V_{1}^{T} \\ V_{2}^{T}\end{array}\right]=U_{1} S V_{1}^{T}=H V_{1}^{T}=\left[\begin{array}{c}H_{1} \\ H_{2}\end{array}\right] V_{1}^{T}$, 
where the diagonal matrix $S>0$, the matrices $\left[\begin{array}{ll}U_{1} & U_{2}\end{array}\right]$ and $\left[\begin{array}{ll}V_{1} & V_{2}\end{array}\right]$ are orthogonal, $H=U_{1} S \in \mathbb{R}^{2 m \times \tilde{n}}$, $H_{1}, H_{2} \in \mathbb{R}^{m \times \tilde{n}}$ and the matrices $H$ and $V_{1}$ have orthogonal columns. Then, the matrix $H_{1}^{T} H_{2}$ can be further decomposed by SVD as $H_{1}^{T} H_{2}=\hat{U} \hat{S} \hat{V}^{T}=$ $\hat{U}\left[\begin{array}{ll}S_{1} & 0 \\ 0 & 0\end{array}\right]\left[\begin{array}{c}\hat{V}_{1}^{T} \\ \hat{V}_{2}^{T}\end{array}\right]$, where $\hat{U}, \hat{V}=\left[\begin{array}{ll}\hat{V}_{1} & \hat{V}_{2}\end{array}\right]^{T} \in \mathbb{R}^{\tilde{n} \times \tilde{n}}$ are orthogonal matrices, $\hat{V}_{2} \in \mathbb{R}^{\tilde{n} \times \tilde{n}}$ and the diagonal matrix $S_{1}>0$. Also, define $F=H_{1} \hat{V}_{2} \in \mathbb{R}^{m \times \tilde{n}}$ and $N_{f}=P_{s}(0)-P_{s}(0) F\left(F^{T} P_{s}(0) F\right)^{-1} F^{T} P_{s}(0)$, where $P_{s}(s)$ is an SNI controller. When $P_{2} \neq 0$, define $N_{2}=P_{s}(0)-P_{s}(0) J\left(J^{T} P_{s}(0) J\right)^{-1} J^{T} P_{s}(0)$, where $J$ is a full column rank matrix satisfying $J J^{T}=P_{2}$. When $P_{2}=0$ and $P_{1} \neq 0, P_{1}$ can be decomposed by $\mathrm{SVD}$ as $P_{1}=\left[\begin{array}{cc}\tilde{U}_{1} & \tilde{U}_{2}\end{array}\right]\left[\begin{array}{cc}S_{2} & 0 \\ 0 & 0\end{array}\right]\left[\begin{array}{c}V_{1}^{T} \\ V_{2}^{T}\end{array}\right]=F_{1} V_{1}^{T}$, where $\left[\begin{array}{ll}\tilde{U}_{1} & \tilde{U}_{2}\end{array}\right]$ and $\left[\begin{array}{ll}V_{1} & V_{2}\end{array}\right]$ are orthogonal matrices, the diagonal matrix $S_{2}>0$ and $F_{1}$ and $V_{1}$ have orthogonal columns. Then, define $N_{1}=$ $P_{s}(0)-P_{s}(0) F_{1}\left(F_{1}^{T} P_{s}(0) F_{1}\right)^{-1} F_{1}^{T} P_{s}(0)$. Next, internal stability of $\left[P(s), P_{s}(s)\right]$ with free body dynamics can be summarised in the following lemma.

Lemma 14 ([10]) Let $P(s)$ be a strictly proper NI plant and $P_{s}(s)$ be an SNI controller.

(1) Suppose $P_{2} \neq 0, N_{f}$ is sign definite and $F^{T} P_{s}(0) F$ is non-singular. Then, $\left[P(s), P_{s}(s)\right]$ is internally stable if and only if $F^{T} P_{s}(0) F<0$ and either

$$
I-N_{f}^{\frac{1}{2}} P_{0} N_{f}^{\frac{1}{2}}-N_{f}^{\frac{1}{2}} P_{1} J\left(J^{T} J\right)^{-2} J^{T} P_{1}^{T} N_{f}^{\frac{1}{2}}>0
$$

when $N_{f} \geq 0$ or

$$
\operatorname{det}\left(I+\tilde{N}_{f} P_{0} \tilde{N}_{f}+\tilde{N}_{f} P_{1} J\left(J^{T} J\right)^{-2} J^{T} P_{1}^{T} \tilde{N}_{f}\right) \neq 0
$$

when $N_{f} \leq 0$ where $\tilde{N}_{f}=\left(-N_{f}\right)^{\frac{1}{2}}$.

If furthermore $P_{1}=0, N_{2}$ is sign definite and $J^{T} P_{s}(0) J$ is non-singular, the necessary and sufficient conditions for the internal stability of $\left[P(s), P_{s}(s)\right]$ reduce to $J^{T} P_{s}(0) J<0$ and either $I-N_{2}^{\frac{1}{2}} P_{0} N_{2}^{\frac{1}{2}}>0$ when $N_{2} \geq 0$ or $\operatorname{det}\left(I+\tilde{N}_{2} P_{0} \tilde{N}_{2}\right) \neq 0$ when $N_{2} \leq 0$ where $\tilde{N}_{2}=\left(-N_{2}\right)^{\frac{1}{2}}$.

If additionally $\operatorname{Ker}\left(P_{2}\right) \subseteq \operatorname{Ker}\left(P_{0}^{T}\right)$, the necessary and sufficient condition for the internal stability of $\left[P(s), P_{s}(s)\right]$ reduces to $J^{T} P_{s}(0) J<0$. When $P_{2}>0$, the necessary and sufficient condition for the internal stability of $\left[P(s), P_{s}(s)\right]$ reduces to $P_{s}(0)<0$.

(2) Suppose $P_{2}=0, P_{1} \neq 0, N_{1}$ is sign definite and $F_{1}^{T} P_{s}(0) F_{1}$ is non-singular. Then $\left[P(s), P_{s}(s)\right]$ is internally stable if and only if $F_{1}^{T} P_{s}(0) F_{1}<0$ and either $I-N_{1}^{\frac{1}{2}} P_{0} N_{1}^{\frac{1}{2}}>0$ when $N_{1} \geq 0$ or $\operatorname{det}(I+$ $\left.\tilde{N}_{1} P_{0} \tilde{N}_{1}\right) \neq 0$ when $N_{1} \leq 0$ where $\tilde{N}_{1}=\left(-N_{1}\right)^{\frac{1}{2}}$.

If furthermore $\operatorname{Ker}\left(P_{1}^{T}\right) \subseteq \operatorname{Ker}\left(P_{0}^{T}\right)$, the necessary and sufficient condition for the internal stability of $\left[P(s), P_{s}(s)\right]$ reduces to $F_{1}^{T} P_{s}(0) F_{1}<0$. when $P_{1}$ is invertible, the necessary and sufficient condition for the internal stability of $\left[P(s), P_{s}(s)\right]$ reduces to $P_{s}(0)<0$.

Next we present the second main result of this paper with the following notation: $\bar{P}_{2}=\lim _{s \rightarrow 0} s^{2} \bar{P}(s), \bar{P}_{1}=$ $\lim _{s \rightarrow 0} s\left(\bar{P}(s)-\frac{\bar{P}_{2}}{s^{2}}\right)$, and $\bar{P}_{0}=\lim _{s \rightarrow 0}\left(\bar{P}(s)-\frac{\bar{P}_{2}}{s^{2}}-\frac{\bar{P}_{1}}{s}\right)$.

Theorem 15 Given a graph $\mathcal{G}$ with incidence matrix $\mathcal{Q}$, satisfying Assumption 5 and modelling the communication links among multiple strictly proper NI agents $\hat{P}_{i}(s)$ (allowing possible poles at the origin) which are appropriately extended to $P_{i}(s)$ as in Fig. 3, robust output feedback consensus is achieved via the feedback control law in (4) or (5) under any external disturbances $w_{1} \in \operatorname{Im}_{\mathfrak{L}_{2}}\left(\mathcal{Q} \otimes I_{m}\right)$ and $w_{2} \in \mathfrak{L}_{2}$ as well as under any model uncertainty $\Delta_{i}(s), i \in\{1, \cdots, n\}$ satisfying Assumption 6 if and only if the necessary and sufficient conditions in Lemma 14 are satisfied for $\left[\bar{P}(s), \bar{P}_{s}(s)\right]$.

PROOF. Lemma 14 guarantees the internal stability of $\left[\bar{P}(s), \bar{P}_{s}(s)\right]$. Nominal output consensus is then achieved without considering the external disturbances $w_{1}$ and $w_{2}$ via internal stability as discussed in the proof of Theorem 10. Then, similar analysis as in the proof of Theorem 10 guarantees robustness against both external disturbances as well as additive SNI model uncertainty.

One could enquire whether the conditions in Lemma 14 simplify or not in some cases. The answer is positive as we present next.

Theorem 16 Given a graph $\mathcal{G}$ with incidence matrix $\mathcal{Q}$, satisfying Assumption 5 and modelling the communication links among $n_{2}$ strictly proper NI agents $\hat{P}_{i}(s)$ (allowing possible poles at the origin) with double poles at the origin (i.e. no single pole at the origin) and $n_{1}$ (at least 1) agents without free body dynamics (i.e. without poles at the origin) in Fig. 3, a necessary and sufficien$t$ condition for robust output feedback consensus via the feedback control law in (4) or (5) under external disturbances $w_{1} \in \operatorname{Im}_{\mathfrak{L}_{2}}\left(\mathcal{Q} \otimes I_{m}\right)$ and $w_{2} \in \mathfrak{L}_{2}$ and under any model uncertainty satisfying Assumption 6 is

$$
J_{n_{2}}^{T} \mathcal{L}_{e, 11} J_{n_{2}}<0
$$

where $J_{n_{2}} \triangleq \operatorname{diag}_{i=1}^{n_{2}}\left\{J_{2, i}\right\}$ with $J_{2, i}$ being full column rank matrices satisfying $J_{2, i} J_{2, i}^{T}=\lim _{s \rightarrow 0} s^{2} P_{i}(s) \neq 0$ for $n_{2}$ agents and $\mathcal{L}_{e, 11} \in \mathbb{R}^{n_{2} m \times n_{2} m}$ is part of the weighted 
Laplacian matrix constructed as follows:

$\mathcal{L}_{e}=\left[\begin{array}{cc}\mathcal{L}_{e, 11} & \mathcal{L}_{e, 12} \\ \mathcal{L}_{e, 12}^{T} & \mathcal{L}_{e, 22}\end{array}\right]=\chi\left(\mathcal{Q} \otimes I_{m}\right) \operatorname{diag}_{j=1}^{l}\left\{P_{s, j}(0)\right\}\left(\mathcal{Q}^{T} \otimes I_{m}\right) \chi^{T}$

where $\chi$ is a permutation matrix such that

$$
\chi \lim _{s \rightarrow 0} s^{2} \operatorname{diag}_{i=1}^{n}\left\{P_{i}(s)\right\} \chi^{T}=\left[\begin{array}{cc}
\operatorname{diag}_{2}\left\{P_{2, i}\right\} & 0 \\
i=1 & \\
0 & 0_{m n_{1} \times m n_{1}}
\end{array}\right]
$$

and $P_{s, j}(s) \forall j \in\{1, \cdots, l\}$ are SNI compensators.

PROOF. It can be seen that this case corresponds to $\bar{P}_{2} \neq 0, \bar{P}_{1}=0$ and $\operatorname{Ker}\left(\bar{P}_{2}\right) \subseteq \operatorname{Ker}\left(\bar{P}_{0}^{T}\right)$ in Theorem 15. The necessary and sufficient condition in this case is $\bar{J}^{T} \bar{P}_{s}(0) \bar{J}<0$ due to Lemma 14 , where $\bar{P}_{2}=\bar{J} \bar{J}^{T}$ with $\bar{J}$ being full column rank. Since

$$
\begin{aligned}
& \bar{P}_{2}=\lim _{s \rightarrow 0} s^{2} \bar{P}(s) \\
& =\lim _{s \rightarrow 0}\left(\mathcal{Q}^{T} \otimes I_{m}\right) s^{2} \operatorname{diag}_{i=1}^{n}\left\{P_{i}(s)\right\}\left(\mathcal{Q} \otimes I_{m}\right) \\
& =\left(\mathcal{Q}^{T} \otimes I_{m}\right) \chi^{T}\left[\begin{array}{cc}
\operatorname{niag}_{i=1}\left\{P_{2, i}\right\} & 0 \\
0 & 0_{m n_{1} \times m n_{1}}
\end{array}\right] \chi\left(\mathcal{Q} \otimes I_{m}\right) \\
& =\left(\mathcal{Q}^{T} \otimes I_{m}\right) \chi^{T} \underbrace{\left[\begin{array}{c}
n_{2} \\
\operatorname{diag}\left\{J_{2, i}\right\} \\
i=1 \\
0
\end{array}\right]}_{J_{P}} \underbrace{\left[\begin{array}{c}
n_{2} \\
\operatorname{diag}\left\{J_{2, i}^{T}\right\}
\end{array}\right]}_{J_{P}^{T}} \chi\left(\mathcal{Q} \otimes I_{m}\right) \\
& =\bar{J} \bar{J}^{T},
\end{aligned}
$$

where $\chi$ is a permutation matrix which also has a representation of $\chi=\left(\Upsilon \otimes I_{m}\right)$ with $\Upsilon$ also being a permutation matrix, $P_{2, i}=\lim _{s \rightarrow 0} s^{2} P_{i}(s) \neq 0$ for the $n_{2}$ agents $P_{s}(s)$ that have a double pole at the origin and $J_{2, i} J_{2, i}^{T}=P_{2, i}$ where $J_{2, i}$ is full column rank matrix. It can be seen that $J_{P}$ has at least $m$ zero rows due to the existence of at least one $m \times m$ agent without free body dynamics. Thus

$$
\begin{aligned}
\bar{J} & =\left(\mathcal{Q}^{T} \otimes I_{m}\right) \chi^{T} J_{P}=\left(\mathcal{Q}^{T} \Upsilon^{T} \otimes I_{m}\right) J_{P} \\
& =\left(\left[\begin{array}{ll}
\hat{\mathcal{Q}}_{1}^{T} & \hat{\mathcal{Q}}_{2}^{T}
\end{array}\right] \otimes I_{m}\right) J_{P}=\left(\hat{\mathcal{Q}}_{1}^{T} \otimes I_{m}\right) \operatorname{diag}_{i=1}^{n_{2}}\left\{J_{2, i}\right\}^{\prime}
\end{aligned}
$$

where $\hat{\mathcal{Q}}_{1}, \quad \hat{\mathcal{Q}}_{2}$ are row submatrices of $\Upsilon \mathcal{Q}=\left[\begin{array}{l}\hat{\mathcal{Q}}_{1} \\ \hat{\mathcal{Q}}_{2}\end{array}\right]$.

According to Assumption 5, the rank property of $\mathcal{Q}$ and the invertibility of permutation matrix $\Upsilon$, the removal of $\hat{\mathcal{Q}}_{2} \otimes I_{m}$ yields a full column rank of $\hat{\mathcal{Q}}_{1}^{T}$ and thus $\operatorname{rank}(\bar{J})=\operatorname{rank}\left(\operatorname{diag}_{i=1}^{n_{2}}\left\{J_{2, i}\right\}\right)$, which also implies that $\bar{J}$ is full column rank due to the full column rank matrix
$J_{2, i}$. Then, with the notation of $J_{n_{2}} \triangleq \operatorname{diag}_{i=1}^{n_{2}}\left\{J_{2, i}\right\}$,

$$
\begin{aligned}
& \bar{J}^{T} \bar{P}_{s}(0) \bar{J}<0 \\
\Leftrightarrow & {\left[\begin{array}{ll}
J_{n_{2}}^{T} & 0
\end{array}\right] \chi\left(\mathcal{Q} \otimes I_{m}\right) \underset{j=1}{l} \operatorname{diag}_{j=1}^{l}\left\{P_{s, j}(0)\right\}\left(\mathcal{Q}^{T} \otimes I_{m}\right) \chi^{T}\left[\begin{array}{c}
J_{n_{2}} \\
0
\end{array}\right]<0 } \\
\Leftrightarrow & {\left[\begin{array}{ll}
J_{n_{2}}^{T} & 0
\end{array}\right] \mathcal{L}_{e}\left[\begin{array}{c}
J_{n_{2}} \\
0
\end{array}\right]=\left[\begin{array}{ll}
J_{n_{2}}^{T} & 0
\end{array}\right]\left[\begin{array}{cc}
\mathcal{L}_{e, 11} & \mathcal{L}_{e, 12} \\
\mathcal{L}_{e, 12}^{T} & \mathcal{L}_{e, 22}
\end{array}\right]\left[\begin{array}{c}
J_{n_{2}} \\
0
\end{array}\right]<0 } \\
\Leftrightarrow & J_{n_{2}}^{T} \mathcal{L}_{e, 11} J_{n_{2}}<0
\end{aligned}
$$

Similar steps as in the proof of Theorem 10 then shows that robustness against external disturbances as well as additive SNI model uncertainty also holds here.

The above theorem gives a necessary and sufficient condition for robust output feedback consensus directly on the graph information and on the D.C. gain of the SNI controllers. The edge weights (i.e. the D.C. gains of the SNI controllers that are connected with agents that have a double pole at the origin) are important in determining the sign definiteness of $J_{n_{2}}^{T} \mathcal{L}_{e, 11} J_{n_{2}}$, in other words, the internal stability of the networked system. The D.C. gains of the remaining SNI controllers are irrelevant and can be freely chosen as long as they are nonsingular.

Remark 17 When the SNI controllers are homogeneous, the consensus law (4) simplifies to $\boldsymbol{u}=$ $\left(\mathcal{Q} \otimes I_{m}\right)\left(I_{n} \otimes P_{s}(s)\right)\left(\mathcal{Q}^{T} \otimes I_{m}\right) \boldsymbol{y}=\mathcal{L}_{n} \otimes P_{s}(s) \boldsymbol{y}$, or in a distributed manner, $\boldsymbol{u}_{i}=P_{s}(s) \sum_{k=1}^{n} a_{i k}\left(\boldsymbol{y}_{i}-\boldsymbol{y}_{k}\right)$. It can be seen that this captures the main result of [18] in the homogeneous plant case but also generalises the results to the heterogeneous plant case. In the case of heterogeneous SNI controllers, the controller is given by $\boldsymbol{u}=\left(\mathcal{Q} \otimes I_{m}\right) \bar{P}_{s}(s)\left(\mathcal{Q}^{T} \otimes I_{m}\right) \boldsymbol{y}=\overline{\mathcal{L}}_{e}(s) \boldsymbol{y}$, which can be interpreted as a weighted graph $\mathcal{G}$ with the edges weighted by the controller transfer function$s P_{s, j}(s), j=1, \cdots, l$, or in a distributed manner: $\boldsymbol{u}_{i}=\sum_{k=1}^{n} a_{i k} P_{s, j}(s)\left(\boldsymbol{y}_{i}-\boldsymbol{y}_{k}\right)$, where $j$ is the edge connected vertex $i$ and $k$. The above facts give a nice intuitive interpretation and explain why we adopt the incidence matrix for the distributed property rather than the Laplacian matrix as indicated earlier.

\subsection{Illustrative Examples}

Two cases are given to illustrate the main results of this paper, Theorems 10 and 15 respectively. The first case considers multiple NI systems without poles at the origin but allowing the plants to be biproper, while the second case is to show the more general case by including NI systems with poles at the origin but under the requirement of strictly proper plants.

\subsubsection{2 lightly damped and 1 undamped flexible struc- tures}

Fig.2 in [7] depicts a flexible structure that can also be studied in this paper. The dynamics can be expressed 
as: $M_{i} \ddot{\boldsymbol{x}}_{i}+C_{i} \dot{\boldsymbol{x}}_{i}+K_{i} \boldsymbol{x}_{i}=\boldsymbol{u}_{i}, \boldsymbol{y}_{i}=\boldsymbol{x}_{i}, i \in\{1, \cdots, 3\}$, where $\boldsymbol{x}_{i}=\left[\begin{array}{l}x_{i, 1} \\ x_{i, 2}\end{array}\right], \quad \boldsymbol{u}_{i}=\left[\begin{array}{l}u_{i, 1} \\ u_{i, 2}\end{array}\right], M_{i}=\left[\begin{array}{cc}m_{i, 1} & 0 \\ 0 & m_{i, 2}\end{array}\right]$, $C_{i}=\left[\begin{array}{cc}c_{i, 1}+c_{i} & -c_{i} \\ -c_{i} & c_{i, 2}+c_{i}\end{array}\right], K_{i}=\left[\begin{array}{cc}k_{i, 1}+k_{i} & -k_{i} \\ -k_{i} & k_{i, 2}+k_{i}\end{array}\right]$. The undamped flexible structure is given by letting the damped term $C_{i}=0$. The parameters are given as follows:

System 1: $k_{1}=k_{1,1}=k_{1,2}=0.5, c_{1}=c_{1,1}=$ $c_{1,2}=0.2, m_{1,1}=m_{1,2}=1$ with initial condition of $\left[\begin{array}{llll}0.5 & 0.1 & 1 & 0.2\end{array}\right]^{T}$

System 2: $k_{2}=k_{2,1}=k_{2,2}=1, c_{2}=c_{2,1}=c_{2,2}=$ 0.1, $m_{2,1}=1, m_{2,2}=0.5$ with initial condition of $\left[\begin{array}{llll}1 & 0.1 & 1.5 & 0.2\end{array}\right]^{T}$

System 3: $k_{3}=k_{3,1}=k_{3,2}=1, c_{3}=c_{3,1}=c_{3,2}=0$, $m_{3,1}=1, m_{3,2}=0.5$ with initial condition of $\left[\begin{array}{llll}1.5 & 0.1 & 2 & 0.2\end{array}\right]^{T}$.

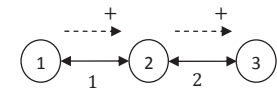

$\mathcal{G}$

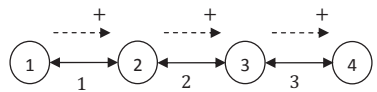

$\mathcal{G}$
Fig. 5. Graph for 3 and 4 NI systems

The communication topology is given in Fig. 5 and thus $\mathcal{Q}=\left[\begin{array}{cc}1 & 0 \\ -1 & 1 \\ 0 & -1\end{array}\right]$ and $\mathcal{L}_{n}=\left[\begin{array}{ccc}1 & -1 & 0 \\ -1 & 2 & -1 \\ 0 & -1 & 1\end{array}\right]$. It can easily be seen that $\max _{i=1}^{3} \bar{\lambda}\left(P_{i}(0)\right)=2>0$. Both the SNI controllers are chosen as $\frac{1}{s+8}$ with an initial condition of -1 such that $\bar{\lambda}\left(P_{i}(0)\right) \bar{\lambda}\left(P_{s, j}(0)\right)=2 * \frac{1}{8}=\frac{1}{4}<$ $\frac{1}{3}=\frac{1}{\lambda\left(\mathcal{L}_{n}\right)} \forall i \in\{1, \cdots, n\}, j \in\{1, \cdots, l\}$. In addition, $\forall i \in\{1, \cdots, n\}, j \in\{1, \cdots, l\}, P_{i}(\infty) P_{s, j}(\infty)=0$, $P_{s, j}(\infty)=0$, which all satisfy the suppositions of Theorem 10. First, without disturbances and model uncertainty, nominal output feedback consensus is achieved via the control law in (4) or (5) as shown in the top two figures of Fig. 6. Then introducing additive SNI model uncertainty, for example given by $\frac{1}{s+4}$ as well as $\mathfrak{L}_{2}$ external disturbances, robust output feedback consensus is also achieved as shown in the bottom two figures of Fig. 6 .

\subsubsection{1 single integrator, 1 double integrator, 1 un- damped and 1 lightly damped flexible structure}

In order to illustrate Theorem 15, systems with free body dynamics will be included in this example. Therefore, a complicated case containing 1 single integrator, 1 double integrator, 1 MIMO undamped and 1 MIMO lightly damped flexible structure is considered in this example. For consistency of dimension, the single integrator and
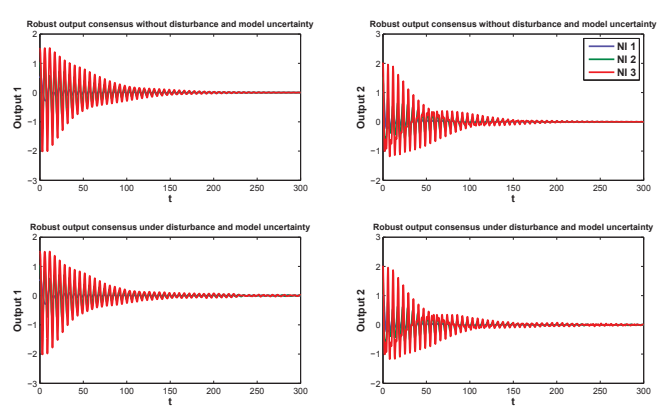

Fig. 6. Robust output consensus of heterogeneous NI systems

the double integrator are extended as follows: $\operatorname{diag}\left\{\frac{1}{s}, 0\right\}$ and $\operatorname{diag}\left\{0, \frac{1}{s^{2}}\right\}$, which also means that the output of single integrator will be coordinated with first outputs of both the undamped and the lightly damped flexible structures, while the output of double integrator will be coordinated with second outputs of both the undamped and the lightly damped flexible structures. The parameters of all NI systems are as follows:

System 1: $\frac{1}{s^{2}}$ with initial condition of $\left[\begin{array}{ll}1 & 0.1\end{array}\right]^{T}$;

System 2: $\frac{1}{s}$ with initial condition of 2;

System 3: $k_{3}=k_{3,1}=k_{3,2}=1, c_{3}=c_{3,1}=c_{3,2}=0$, $m_{3,1}=1, m_{3,2}=0.5$ with initial condition of $\left[\begin{array}{llll}3 & 0.1 & 3 & 0.2\end{array}\right]^{T}$;

System 4: $k_{4}=k_{4,1}=k_{4,2}=1, c_{4}=c_{4,1}=c_{4,2}=$ $0.1, m_{4,1}=1, m_{4,2}=0.5$ with initial condition of $\left[\begin{array}{llll}4 & 0.1 & 4 & 0.2\end{array}\right]^{T}$.

The communication topology is given in Fig. 5 and thus $\mathcal{Q}=\left[\begin{array}{ccc}1 & 0 & 0 \\ -1 & 1 & 0 \\ 0 & -1 & 1 \\ 0 & 0 & -1\end{array}\right]$ and $\mathcal{L}_{n}=\left[\begin{array}{cccc}1 & -1 & 0 & 0 \\ -1 & 2 & -1 & 0 \\ 0 & -1 & 2 & 1 \\ 0 & 0 & -1 & 1\end{array}\right]$. Al1 three SNI controllers are chosen as $-\frac{s+1}{s+2}$ with an initial condition of 0.1 . Through the calculation process discussed earlier in subsection 2.2, the inequality condition (9) can be verified as $\operatorname{det}\left(I+\tilde{N}_{f} \bar{P}_{0} \tilde{N}_{f}+\right.$ $\left.\tilde{N}_{f} \bar{P}_{1} J\left(J^{T} J\right)^{-2} J^{T} \bar{P}_{1}^{T} \tilde{N}_{f}\right)=3.7813 \neq 0$, which indicates internal stability of $\left[\bar{P}(s), \bar{P}_{s}(s)\right]$. Firstly, without disturbances and model uncertainty, nominal output feedback consensus is achieved via the output feedback control law (4) or (5) as shown in the top two figures of Fig. 7. If the same external disturbances and model uncertainty as in subsection 2.3.1 are inserted, robust output feedback consensus is also achieved via output feedback control law (4) or (5) as shown in the bottom two figures of Fig. 7. The left two figures of Fig. 7 indicate the output of the single integrator (System 2) is coordinated with the first output of undamped flexible structure (System 3) and the first output of lightly damped flexible structure (System 4) even under exter- 
nal disturbances and model uncertainty. Similarly, the right two figures of Fig. 7 indicate the output of the double integrator (System 1) is coordinated with the second output of undamped flexible structure (System 3) and the second output of the lightly damped flexible structure (System 4) even under external disturbances and model uncertainty.
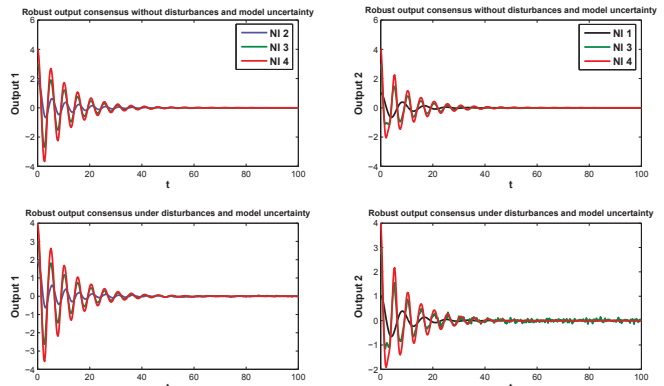

Fig. 7. Robust output consensus of heterogeneous NI systems

\section{Extension to Robust Cooperative Control}

In this section, we exploit the proposed consensus results for cooperative tracking to obtain a robust cooperative control framework for multiple NI systems. An famous rendezvous problem is presented to show the effectiveness of the proposed framework. Other cooperative problems can be obtained analogously by adapting the proposed consensus algorithm. The objective of cooperative tracking is to achieve the convergence of all agents' outputs to a pre-defined constant reference while the objective of rendezvous is its direct application for all the agents to converge to a pre-defined point. To solve this problem, let us first define a matrix $\mathcal{B}$ to express the connections between agents following reference [11]: $\mathcal{B}=\operatorname{diag}_{i=1}^{n}\left\{b_{i}\right\}$, where $b_{i}=1$ if agent $i$ is connected with a reference, otherwise $b_{i}=0$. From $\mathcal{L}_{n}=Q Q^{T}$, we can similarly decompose $\mathcal{B}$ as $\mathcal{Q}_{b} \mathcal{Q}_{b}^{T}$ where $\mathcal{Q}_{b}$ is full column rank matrix. It can be seen that $\mathcal{Q}_{b}$ is defined analogously as $\mathcal{Q}$ by $\mathcal{Q}_{b}=\left[\beta_{1}, \cdots, \beta_{l_{b}}\right]$, where $l_{b}$ is the number of agents connected to the reference and $\beta_{j_{b}} \forall j_{b} \in$ $\left\{1, \cdots, l_{b}\right\}$ is a vector in $\mathbb{R}^{n}$ with the $i$ th element being a 1 if agent $i$ is connected to the reference. Then, the incidence matrix $\mathcal{Q}$ can be augmented with $\mathcal{Q}_{b}$ to give $\left[\mathcal{Q} \mathcal{Q}_{b}\right]$ which also shows the additional links from the reference to agents. The augmented matrix $\left[\mathcal{Q} \mathcal{Q}_{b}\right]$ still guarantees that $\left(\left[\begin{array}{ll}\mathcal{Q} & \mathcal{Q}_{b}\end{array}\right]^{T} \otimes I_{m}\right) \bar{P}(s)\left(\left[\begin{array}{ll}\mathcal{Q} & \mathcal{Q}_{b}\end{array}\right] \otimes I_{m}\right)$ is NI in Fig. 2 and thus NI system theory can be applied to the robust output feedback consensus problem as shown in the previous section. The main result of this section is given next.

Theorem 18 Given a graph $\mathcal{G}$ with incidence matrix $\mathcal{Q}$, satisfying Assumption 5 and modelling the communication links among multiple NI agents $\hat{P}_{i}(s)$ which are appropriately extended to $P_{i}(s)$ as in Fig. 3, robust cooperative output tracking of a constant reference $\boldsymbol{r}$ is achieved via the output feedback control law

$\boldsymbol{u}_{c t}=\left(\left[\begin{array}{ll}\mathcal{Q} & \mathcal{Q}_{b}\end{array}\right] \otimes I_{m}\right) \bar{P}_{s}(s)\left(\left[\begin{array}{ll}\mathcal{Q} & \mathcal{Q}_{b}\end{array}\right]^{T} \otimes I_{m}\right)\left(\boldsymbol{y}-\mathbf{1}_{n} \otimes \boldsymbol{r}\right)$,

or in a distributed manner $\forall i \in\{1, \cdots, n\}$ by

$$
\boldsymbol{u}_{i}^{c t}=\sum_{k=1}^{n} a_{i k} P_{s, j}(s)\left(\boldsymbol{y}_{i}-\boldsymbol{y}_{k}\right)+b_{i} P_{s, j_{b}}(s)\left(\boldsymbol{y}_{i}-\boldsymbol{r}\right)
$$

where $j \in\{1, \cdots, l\}$ corresponds to the edge number connecting agents $i$ and $k$, and $j_{b} \in\left\{1, \cdots, l_{b}\right\}$ corresponds to the link number connecting reference $\boldsymbol{r}$ to agent $i$, under any external disturbances $w_{1} \in \operatorname{Im}_{\mathfrak{L}_{2}}\left(\mathcal{Q} \otimes I_{m}\right)$ and $w_{2} \in \mathfrak{L}_{2}$ as well as under any model uncertainty satisfying Assumption 6 if and only if the relevant conditions in Theorem 10 or Lemma 14 are satisfied for $\left[\bar{P}(s), \bar{P}_{s}(s)\right]$.

PROOF. Given a constant reference $\boldsymbol{r}$, the matrix $\left[\begin{array}{ll}\mathcal{Q} & \mathcal{Q}_{b}\end{array}\right]^{T}$ has the property that

$$
\overline{\boldsymbol{y}}=\left(\left[\begin{array}{c}
\mathcal{Q}^{T} \\
\mathcal{Q}_{b}^{T}
\end{array}\right] \otimes I_{m}\right)\left(\boldsymbol{y}-\mathbf{1}_{n} \otimes \boldsymbol{r}\right)=\left[\begin{array}{c}
\left(\mathcal{Q}^{T} \otimes I_{m}\right) \boldsymbol{y} \\
\left(\mathcal{Q}_{b}^{T} \otimes I_{m}\right)\left(\boldsymbol{y}-\mathbf{1}_{n} \otimes \boldsymbol{r}\right)
\end{array}\right]
$$

since $\left(\mathcal{Q}^{T} \otimes I_{m}\right)\left(\mathbf{1}_{n} \otimes \boldsymbol{r}\right)=\mathbf{0}$ due to the null space property of incidence matrix in Section 1. Therefore, internal stability guaranteed by Theorem 10 or Lemma 14 leads to $\overline{\boldsymbol{y}} \rightarrow \mathbf{0}$, which is equivalent to $\boldsymbol{y}_{i} \rightarrow \boldsymbol{y}_{k} \forall i \neq$ $k \in\{1, \cdots, n\}$ due to $\left(\mathcal{Q}^{T} \otimes I_{m}\right) \boldsymbol{y} \rightarrow \mathbf{0}$ and $\boldsymbol{y}_{j_{b}} \rightarrow$ $\boldsymbol{r} \forall j_{b} \in\left\{1, \cdots, l_{b}\right\}$ due to $\left(\mathcal{Q}_{b}^{T} \otimes I_{m}\right)\left(\boldsymbol{y}-\mathbf{1}_{n} \otimes \boldsymbol{r}\right) \rightarrow \mathbf{0}$. This then implies robust cooperative output tracking is achieved, i.e., $\boldsymbol{y}_{i}=\boldsymbol{y}_{k}=\boldsymbol{r} \forall i \in\{1, \cdots, n\}$ and $k \in\{1, \cdots, n\} /\{i\}$ via arguments like those in the proof of Theorem 10 .

\subsection{D Rendezvous Missions}

A direct application of cooperative tracking, robust rendezvous is shown in a $3 \mathrm{D}$ scenario. We use plants composed of $\frac{1}{s^{2}} I_{3}, \frac{1}{s^{2}} I_{3}, \frac{1}{s} I_{3}$ with the initial conditions in 3 axis being $\left[\begin{array}{lllllll}1 & 0.1 & 1 & 1 & 1 & -0.1\end{array}\right]^{T}$, $\left[\begin{array}{llllll}2 & 0.2 & 2 & -1 & 2 & -0.2\end{array}\right]^{T}$ and $\left[\begin{array}{llll}0.3 & 0.5 & 0.8\end{array}\right]^{T}$ respectively. The pre-defined rendezvous point is $\left[\begin{array}{lll}1 & -0.5 & 0\end{array}\right]^{T}$. The graph is exactly the same as Fig. 5 and the reference only sends information to agent 3 , which gives $\mathcal{B}=\operatorname{diag}\{0,0,1\}$ and $\mathcal{Q}_{b}=\left[\begin{array}{lll}0 & 0 & 1\end{array}\right]^{T}$. Comparing with the cases in Theorem 15, it can be seen that this is 
the case when $P_{1} \neq 0$ and $P_{2} \neq 0$. Therefore, Lemma 14 is used and it is easy to see that $N_{f} \leq 0$ and $\operatorname{det}\left(I+\tilde{N}_{f} P_{0} \tilde{N}_{f}+\tilde{N}_{f} P_{1} J\left(J^{T} J\right)^{-2} J^{T} P_{1}^{T} \tilde{N}_{f}\right)=1 \neq 0$. Robust rendezvous is then guaranteed via the cooperative tracking controller (10) or (11) in Theorem 18. It can be seen from Fig. 8 that the cooperative tracking control law (10) or (11) is able to drive the agents from the initial positions marked as diamonds to the final rendezvous point even under the same external disturbances and model uncertainty as in subsection 2.3.1.

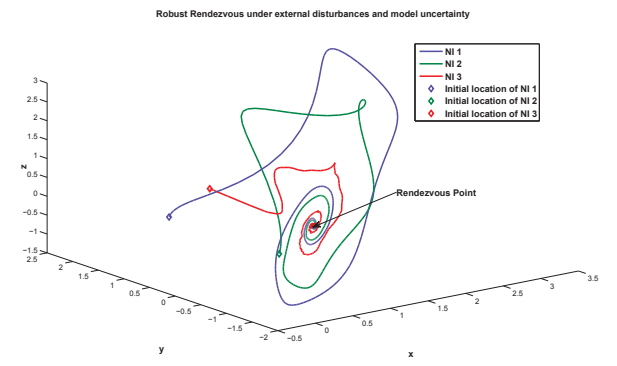

Fig. 8. 3D robust rendezvous of heterogeneous NI systems

\section{Conclusion Remarks}

Robust cooperative control for heterogeneous NI systems is proposed via NI systems theory. Robust output feedback consensus against external disturbances and NI model uncertainty is studied first and then the result$\mathrm{s}$ are exploited for cooperative tracking to derive a cooperative control framework. The key contributions of this paper can be summarised as: (1) cooperative control which is robust to exogenous disturbances and SNI model uncertainty for general heterogeneous network of MIMO NI systems under any undirected and connected graph; (2) only exploiting output feedback information in contrast to full state information commonly used in the literature; (3) providing a whole class of cooperative control laws, i.e. SNI controllers, that can be tuned for performance and characterising conditions that can be easily checked for robust output feedback consensus; (4) showing how consensus and cooperative control problems can exploit powerful internal stability and robust stability results available in the literature.

\section{References}

[1] R.B. Bapat. Graph and Matrices. Springer, Springer London Dordrecht Heidelberg New York, 2010.

[2] C.H. Cai and G. Hagen. Stability analysis for a string of coupled stable subsystems with negative imaginary frequency response. IEEE Trans. Autom. Control, 55:1958-1963, 2010.

[3] A. Ferrante, A. Lanzon, and L. Ntogramatzidis. Foundations of not necessarily rational negative imaginary systems theory: Relations between classes of negative imaginary and positive real systems. IEEE Trans. Autom. Control, submitted in 2014. See also technical report at arXiv:1412.5709

[4] A. Ferrante and L. Ntogramatzidis. Some new results in the theory of negative imaginary systems with symmetric transfer matrix function. Automatica, 49:2138-2144, 2013.
[5] G.H. Golub and C.F. Van Loan. Matrix Computations. JHU Press, Baltimore, MD, USA, 2012.

[6] R. Grone and R. Merris. The laplacian spectrum of a graph ii. SIAM Journal on Discrete Mathematics, 22:221-229, 1994.

[7] A. Lanzon and I.R. Petersen. Stability robustness of a feedback interconnection of systems with negative imaginary frequency response. IEEE Trans. Autom. Control, 53:10421046, 2008.

[8] Z.K. Li, Z.S. Duan, G.R. Chen, and L. Huang. Consensus of multiagent systems and synchronization of complex networks: A unified view point. IEEE Trans. on Circuits and Systems, 57:213-224, 2010.

[9] M. A. Mabrok, A. G. Kallapur, I.R. Petersen, and A. Lanzon. Spectral conditions for negative imaginary systems with applications to nano-positioning. IEEE/ASME Transactions on Mechatronics, 19:895-903, 2014.

[10] M.A. Mabrok, A.G. Kallapur, I.R. Petersen, and A. Lanzon. Generalizing negative imaginary systems theory to include free body dynamics: Control of highly resonant structures with free body motion. IEEE Trans. Autom. Control, 59:2692-2707, 2014.

[11] W. Ni and D.Z. Cheng. Leader-following consensus of multiagent systems under fixed and switching topologies. Systems and Control Letters, 59:209-217, 2010.

[12] M.R. Opmeer. Infinite-dimensional negative imaginary systems. IEEE Trans. Autom. Control, 56:2973-2976, 2011.

[13] I.R. Petersen and A. Lanzon. Feedback control of negativeimaginary systems. IEEE Control Systems Magazine, 30:5472, 2010.

[14] W. Ren and R. W. Beard. Distributed Consensus in Multivehicle Cooperative Control. Communications and Control Engineering Serie, Springer-Verlag, London, 2008.

[15] Z. Song, A. Lanzon, S. Patra, and I. R. Petersen. Robust performance analysis for uncertain negative-imaginary systems. International Journal of Robust and Nonlinear Control, 22(3):262-281, Feb. 2012.

[16] Z. Song, A. Lanzon, S. Patra, and I.R. Petersen. A negative-imaginary lemma without minimality assumptions and robust state-feedback synthesis for uncertain negativeimaginary systems. Systems and Control Letters, 61:12691276, 2012.

[17] Y.F. Su and J. Huang. Cooperative robust output regulation of a class of heterogeneous linear uncertain multi-agent systems. International Journal of Robust and Nonlinear Control, 2013, DOI: 10.1002/rnc.3027.

[18] J.N. Wang, A. Lanzon, and I.R. Petersen. A robust output feedback consensus protocol for networked negativeimaginary systems. 2014 IFAC World Congress, Cape Town, South Africa, Aug. 2014.

[19] J.N. Wang, A. Lanzon, and I.R. Petersen. Robust output feedback consensus for networked negative-imaginary systems. IEEE Trans. Autom. Control, in press, DOI:10.1109/TAC.2015.2395472.

[20] J.L. Xiong, I.R. Petersen, and A. Lanzon. A negative imaginary lemma and the stability of interconnections of linear negative imaginary systems. IEEE Trans. Autom. Control, 55:2342-2347, 2010.

[21] K. Zhou, J.C. Doyle, and K. Glover. Robust and Optimal Control. NJ: Prentice-Hall, Englewood Cliffs, 1996.

[22] L.J. Zhu and Z.Y. Chen. Robust homogenization and consensus of nonlinear multi-agent systems. Systems and Control Letters, 65:50-55, 2014. 